\title{
ELECTRICAL PROPERTIES OF LEAD PHTHALOCYANINE FILMS
}

\author{
N. Abd El-Renim and A.E. El-Samahy \\ Physics Department, Faculty of Science, Alexandria University \\ Alexandria, Egypt
}

(Received Oclober 27, 1995; revised version April 23, 1996)

\begin{abstract}
Current density-voltage characteristics have been obtained from thin films of lead phthalocyanine particles dispersed in a polymer binder when sandwiched between olımic gold and blocking aluminium electrodes. At low voltages, the current in the forward direction shows Schottky diode behaviour. The diode parameters are evaluated using the model of Cheung and Cheung. Barrier heights and widtlis are determined as a function of applied voltage. A number of parameters is evaluated on the basis of the theory of space-charge-limited conduction, and the following values are obtained: $p_{0}=8.5 \times 10^{18} \mathrm{~m}^{-3}$, concentration of the traps per unit energy range at the valence band $P_{0} \approx 2.5 \times 10^{44} \mathrm{~J}^{-1} \mathrm{~m}^{-3}$, temperature parameter of trapping distribution $T_{c} \approx 500 \mathrm{~K}$ and total trapping concentration, $N_{t} \approx 1.7 \times 10^{24} \mathrm{~m}^{-3}$.
\end{abstract}

PACS numbers: $72.20 .-\mathrm{i}$

\section{Introduction}

Most investigations [1-3] of the electrical conduction properties of phthalocyanines have been carried out on thin evaporated films which are microcrystalline in nature. Other workers [4-6] have also found that a dispersion of phthalocyanine particles in appropriate selection of polymer binder could improve the cell performance. The nature of the electronic process in phthalocyanine is generally influenced by the presence of traps [7]. The phthalocyanines that have attracted most interest to date are metal free phthalocyanine and its metal derivative copper phthalocyanine $(\mathrm{CuPc})$. Lead phthalocyanine $(\mathrm{PbPc})$ has received considerable less attention. $\mathrm{PbPc}$ is a $p$-type semiconductor and a metal such as gold is required to provide ohmic contact [1]. Other electrodes such as $\mathrm{Al}$ and In are found to form blocking contacts [8]. Additionally, it is believed that sandwiching an organic semiconductor between an ohmic and a blocking contact should lead to the Schottky diode behaviour and that the rectification behaviour is increased considerably by the presence of oxygen [9] which increases the acceptor concentration. 
The object of this paper is to study the current density voltage characteristics for thin films of PbPc particles dispersed in a polymer binder, polyvinylacetate (PVA), using ohmic gold and blocking aluminium electrodes.

\section{Experimental details}

The organic semiconductor used in the present study is the $\beta$-form of lead phthalocyanine ( $\mathrm{PbPc})$. Films of PbPc-PVA prepared by solvent coating, a dispersion of $\mathrm{PbPc}$ particles in polyvinylacetate (PVA), a polymer obtained from Aldrich Chemical Company Inc. The fabrication of the cells, $\mathrm{Al}-\mathrm{PbPc}-\mathrm{Au}$ was similar to that reported previously [0]. All cells contained approximately $60 \%$ of $\mathrm{PbPc}$ dispersed in the polymer binder PVA, and their thickness ranged from 2 to $4 \mu \mathrm{m}$. Sample currents were measured using a stabilized power supply and a Keithley $610 \mathrm{C}$ electrometer. Forward bias is defined with the gold electrode biased positively. The temperature of the sample was measured directly by means of $\mathrm{Ni}-\mathrm{Cr} / \mathrm{Ni}-\mathrm{Al}$ thermocouple connected to a Keithley 871 digital thermometer.

\section{Results and discussion}

The forward and reverse current density-voltage $(J-V)$ characteristics at room temperature, for the cell Al/PbPc-PVA/Au are shown in Fig. 1. It is clear that the current density and the shape of the $J-V$ curve are dependent on the bias direction.

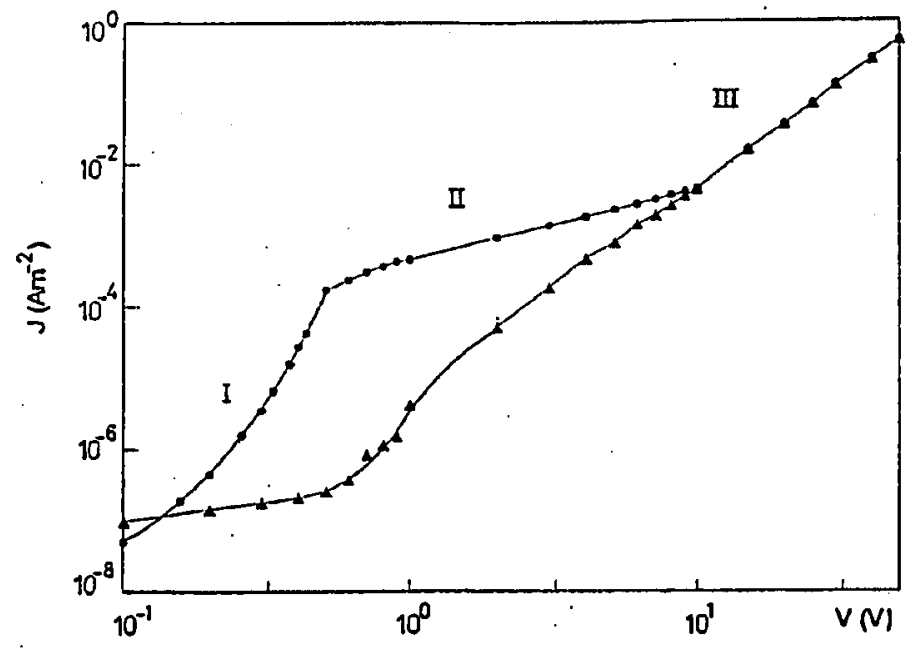

Fig. 1. Typical $J-V$ characteristics for Al/PbPc-PVA/Au sample. - forward bias, $\Delta$ reverse bias. Temperature $293 \mathrm{~K}$. Thickness $2 \mu \mathrm{m}$.

The forward bias characteristic exhibits three different regions. At low voltages the current density varies exponentially with voltage as shown in Fig. 2. This behaviour accorded with the thermoionic emission function 


$$
J=J_{\mathbf{s}}\left[\exp \left(\frac{e V_{\mathrm{a}}}{k T}\right)-1\right]
$$

where $e$ is the electronic charge, $V_{\mathrm{a}}$ is the applied voltage across the diode, $k$ is the Boltzmann constant, $T$ is the absolute temperature and $J_{\mathrm{s}}$ is the reverse saturation current density represented by

$$
J_{\mathrm{s}}=A^{* *} T^{2} \exp \left(\frac{-e \phi_{\mathrm{b}}}{k T}\right),
$$

where $A^{* *}$ is the effective Richardson constant and $\phi_{\mathrm{b}}$ is the Schottky barrier height of the diode. The value of the barrier height can be evaluated by making the $\ln J$ versus $V$ plot. For voltages larger than a few $k T / e$, this plot will be a straight line whose extrapolated intercept with the vertical axis gives $J_{\mathrm{s}}$ from which $\phi_{\mathrm{b}}$ can be calculated. Ilowever, departure from the ideal thermoionic emission theory has been reported for practical Schottky barrier, that is, the forward $J-V$ characteristic is expressed as [10]

$$
J=J_{\mathrm{s}}\left[\exp \left(\frac{e V}{n k T}\right)-1\right],
$$

where $n$ is called the ideality factor.

If the diode has a series resistance $R$, the straight line part of the plot will then be confined to the voltage interval $k T / e \ll V<$ departure point from the straight line due to the voltage drop across the resistance $R$. For large values of $R$, this interval will be too small to get a reliable value of $\phi_{\mathrm{b}}$. Norde [11] has developed a method capable of determining $\phi_{\mathrm{b}}$ and $R$ for ideal Schottky diodes (i.e. $n=1$ ). Sato and Yasumura [12] modified Norde's approach to evaluate $\phi_{\mathrm{b}}$, $n$ and $R$ from the forward $J-V$ data at two different temperatures. Cheung and Cheung [13] developed another method to evaluate the values of diode parameters $\phi_{\mathrm{b}}, n$ and $R$ from a single forward $J-V$ data. The voltage $V_{\mathrm{a}}$ across the diode can be expressed in terms of the voltage drop across the series combination of the diode and the resistor $R$, thus $V_{\mathrm{a}}=V-J A_{\mathrm{e}} R$, where $A_{\mathrm{e}}$ is the effective area of the device, and for $V_{\mathrm{a}} \gg k T / e, \mathrm{Eq}$. (3) becomes

$$
J=J_{\mathrm{s}} \exp \left(\frac{V-J A_{\mathrm{e}} R}{n k T}\right) .
$$

Figure 2a shows a semi-logarithmic plot of the forward biased $J-V$ characteristic below $800 \mathrm{mV}$. It can be seen from the figure that only a few points in the low voltage region are in good agreement with the theory predicted by Eq. (3) and yield $n=1.4$ and $\phi_{\mathrm{b}}=1.18 \mathrm{eV}$. The precise diode parameters $n, \phi_{\mathrm{b}}$ and $R$ were evaluated using Cheung and Cheung model [13]. Figure 2b shows a good agreement between the experimental values and those derived using the Cheung and Cheung model. The parameters used for the curve fitting were determined as described below. Equation (4) can be rewritten in terms of $J$ as

$$
V=R A_{\mathrm{e}} J+n \phi_{\mathrm{b}}+\left(\frac{n k T}{e}\right) \ln \left(\frac{J}{A^{* *} T^{2}}\right) .
$$

Differentiating with respect to $J$ and rearranging terms gives

$$
\frac{\mathrm{d} V}{\mathrm{~d} \ln J}=R A_{\mathrm{e}} J+\frac{n k T}{e} \text {. }
$$



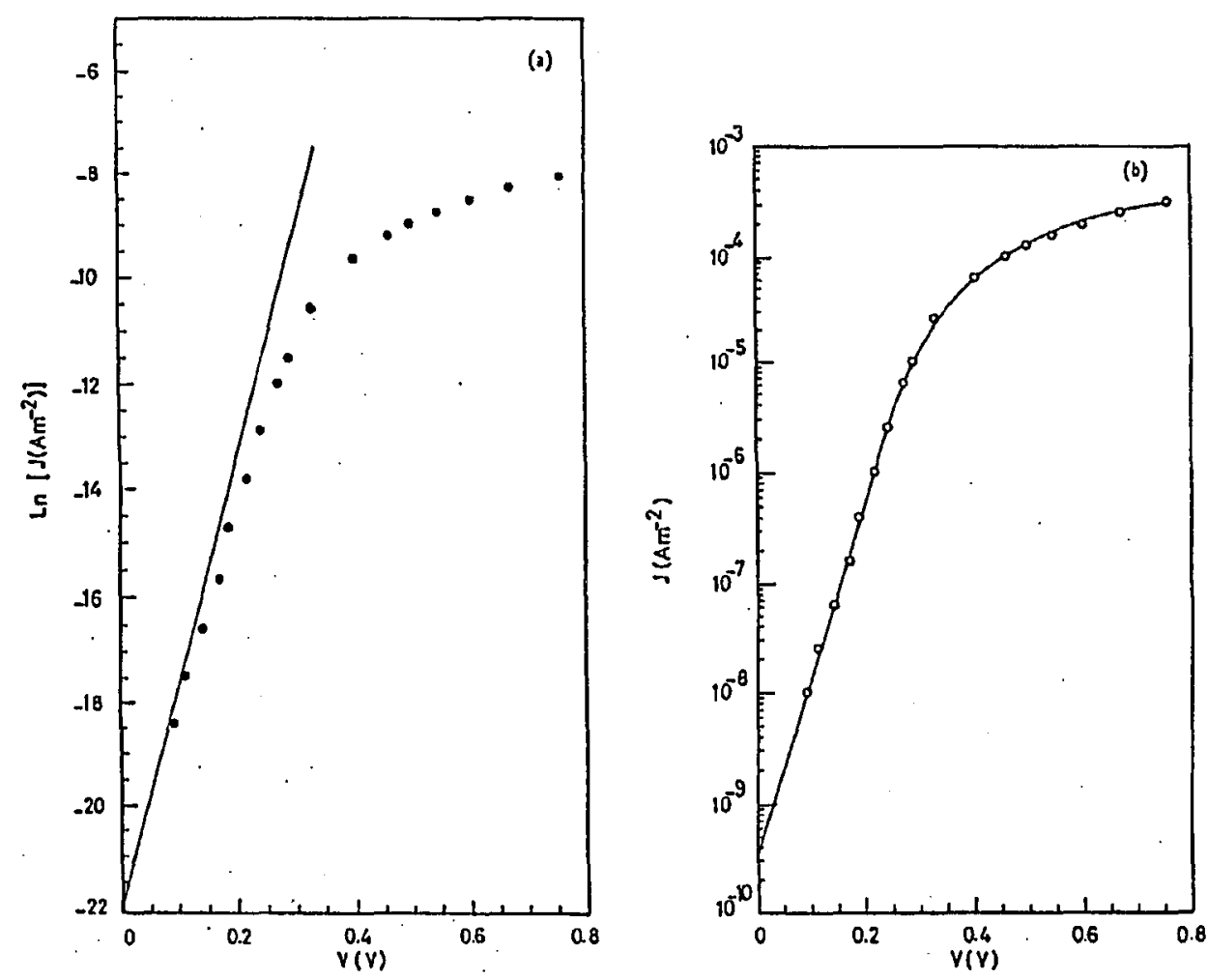

Fig. 2. (a) Dependence of current density $J$ on $V$ for forward biased. - experimental, - theoretical. (b) Forward ln $J-V$ characteristics. - theoretical curve fitting (Cheung-Cheung model), o experimental.

It is evident from the above equation that if one plots $\mathrm{d} V / \mathrm{d} \ln J$ versus $J$, the slope of the line thus obtained equals $R A_{\mathrm{e}}$, while its intersection with the ordinate axis $n k T / e$. This is shown in Fig. 3, and clearly yields linear behaviour. The derived values of the parameters are $n=1.3$ and $R=12.2 \mathrm{M} \Omega$.

To evaluate the barrier height $\phi_{\mathrm{b}}$, Cheung and Cheung proposed the following function:

$$
H(J)=V-\left(\frac{n k T}{e}\right) \ln \left(\frac{J}{A^{* *} T^{2}}\right) .
$$

Comparison of (7) and (5) gives Cheung and Cheung equation

$$
H(J)=R A_{\mathrm{e}} J+n \phi_{\mathrm{b}} \text {. }
$$

A plot of $H(J)$ versus $J$ gives a straight line with a slope $R A_{\mathrm{e}}$, and intercept on the $y$ axis equals to $n \phi_{\mathrm{b}} ;$ Fig. 4 shows $H(J)$ versus $J$. The derived values of the parameters are $R=12.3 \mathrm{M} \Omega$ and $\phi_{\mathrm{b}}=1.12 \mathrm{eV}$. The derived values of $n=1.3$ and $\phi_{\mathrm{b}}=1.12$ are in good agreement with the values deduced from the linear region of $\ln J$ versus $V$ plot. The value of $\phi_{\mathrm{b}}$ is consistent with that for $\mathrm{Au} / \mathrm{PbPc} / \mathrm{Al}$ [1]. 


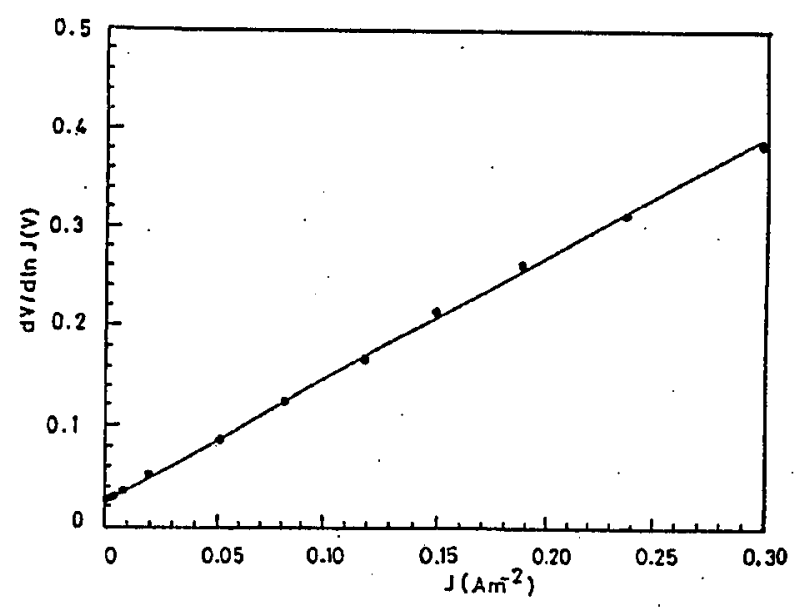

Fig. 3. (dV/d ln $J)$ versus $J$ plot of forward biased Al/PbPc-PVA/Au.

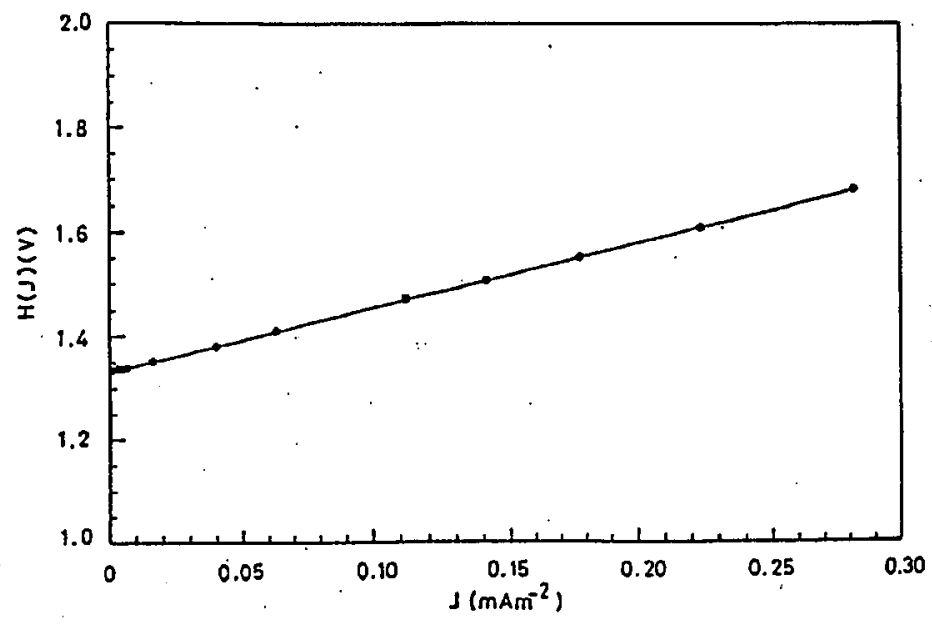

Fig. 4. $H(J)$ versus $J$ plot of forward biased Al/PbPc-PVA/Au.

At higher voltages, above $800 \mathrm{mV}$, there are two distinct regions. The first region has a characteristic slope $\approx 1$, this region is ohmic and it is attributed to thermally generated carriers + electrons. The second region has a power-law in the form $J \sim V^{2.7}$, indicates a space charge limited conduction. The first region adheres to the relation

$$
J=\left(n_{0} e \mu_{\mathrm{e}}+p_{0} e \mu_{\mathrm{p}}\right) \frac{V}{d}
$$

where $p_{0}$ and $n_{0}$ are respectively the concentration of thermally generated holes and electrons, $\mu_{\mathrm{p}}$ and $\mu_{\mathrm{e}}$ are the hole and electron mobilities respectively.

The space-charge limited conduction (SCLC) occurring in the second region may be interpreted in terms of the presence of traps, distributed exponentially 
with energy in the band gap of PbPc. The distribution is given by

$$
P(E)=\frac{P_{0}}{k T_{\mathrm{c}}} \exp \left(-\frac{E}{k T_{\mathrm{c}}}\right),
$$

where $P(E)$ is the trap concentration per unit energy at an energy $E$ above the valence band edge, $P_{0}$ is the value of $P(E)$ at the valence band and $T_{\mathrm{c}}$ is the characteristic temperature which describes the exponential distribution of traps. The total density of traps $N_{t}$ comprising the distribution is given by evaluating $\int P(E) \mathrm{d} E$ from the valence band edge to infinity and yields the result as given by $[14]$

$$
N_{t}=P_{0} k T_{c} \text {. }
$$

The transition or cross-over voltage $V_{\mathrm{t}}$ between ohmic and SCLC is given by [15]

$$
V_{\mathrm{t}}=\left(\frac{P_{0}}{N_{\mathrm{v}}}\right)^{1 / l} \frac{\mathrm{d}^{2} e N_{t}}{\varepsilon}
$$

where $l$ is the ratio $T_{\mathrm{c}} / T$.

According to Lampert [16], the SCLC region with exponential distribution of traps adheres to

$$
J=e \mu_{\mathrm{p}} N_{\mathrm{v}}\left(\frac{\varepsilon}{e N_{t}}\right)^{l} \frac{V^{l+1}}{d^{2 l+1}}
$$

where $\varepsilon$ is the permittivity of $\mathrm{PbPc}, N_{\mathrm{v}}$ is the effective density of states in the valence band and $d$ is the film thickness.

In order to estimate values for the hole trap parameters, it is necessary to assume plausible values for $\mu_{\mathrm{p}}, N_{\mathrm{v}}$ and $\varepsilon$. These values were taken to be similar to those for thin films of $\mathrm{Au}-\mathrm{PbPc}-\mathrm{Au}$ samples [1], and thus $\mu_{\mathrm{p}}=6.05 \times$ $10^{-10} \mathrm{~m}^{2} \mathrm{~V}^{-1} \mathrm{~s}^{-1}, N_{\mathrm{v}}=10^{27} \mathrm{~m}^{-3}$ and $\varepsilon=3.16 \times 10^{-11} \mathrm{~F} \mathrm{~m}^{-1}$ were used. An analysis of the temperature dependence of the $J-V$ curves, Fig. 1 yields a value of thermally generated hole concentration $p_{0}=8.5 \times 10^{18} \mathrm{~m}^{-3}$, which is close in magnitude to that reported in literature, e.g. $5.62 \times 10^{17} \mathrm{~m}^{-3}(\mathrm{Al} / \mathrm{PbPc} / \mathrm{Au})$ [1] and $9.1 \times 10^{17} \mathrm{~m}^{-3}(\mathrm{Au} / \mathrm{CuPc} / \mathrm{Au})[15]$. Equations (5), (6) and (7) yield the following values of trap parameters: $l \approx 1.7, T_{\mathrm{c}} \approx 500 \mathrm{~K}, T=293 \mathrm{~K}, P_{0} \approx 2.5 \times 10^{44} \mathrm{~J}^{-1} \mathrm{~m}^{-3}$ and $N_{t} \approx 1.7 \times 10^{24} \mathrm{~m}^{-3}$.

The trap concentration is a factor somewhat higher than for the value obtained for $\mathrm{Au} / \mathrm{CuPc} / \mathrm{Au}[15]$ and taking into account the difference in material and preparation conditions, are clearly comparable.

The reverse bias $J-V_{r}$ characteristics below region III are shown, in the form of $\ln J$ versus $V_{\mathrm{r}}^{1 / 2}$, in Fig. 5 . The linear portions of the curve may be interpreted in terms of either the Poole-Frenkel eflect (field-assisted thermal detrapping of carriers) or the Schottky effect (field-lowering of the interfacial barrier at the injected electrode interface). The $J-V$ expressions for these processes are given [17] by

$$
J=J_{0} \exp \left(\frac{\beta_{P F} V_{\mathrm{r}}^{1 / 2}}{2 k T w^{1 / 2}}\right)
$$

for the Poole-Frenkel effect, and by

$$
J=A^{* *} T^{2} \exp \left(\frac{-\varphi_{\mathrm{S}}}{k T}\right)\left(\frac{\beta_{\mathrm{S}} V_{\mathrm{r}}^{1 / 2}}{k T w^{1 / 2}}\right)
$$




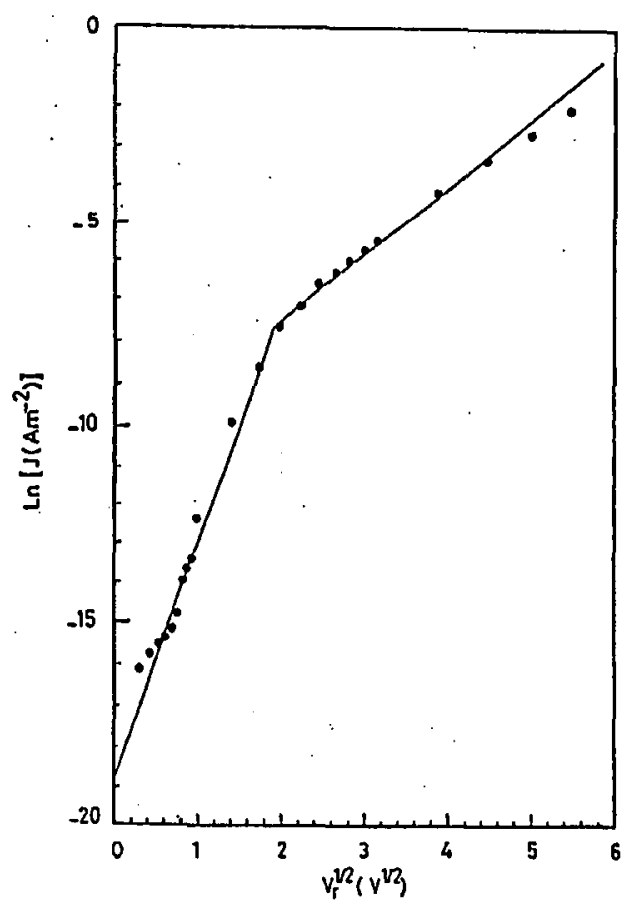

Fig. 5. Reverse bias $J-V$ characteristics for Al/PbPc-PVA/Au sample showing a linear dependence of $\ln J$ on $V_{\mathrm{r}}^{1 / 2}$.

for the Schottky effect, where $\varphi_{S}$ is the Schottky barrier height at the injected electrode interface, $w$ is the width of the Schottky barrier at the injected electrode interface, $J_{0}=\sigma_{0}\left(V_{\mathrm{r}} / w\right)$ is the low-field current density, $\beta_{\mathrm{PF}}$ and $\beta_{\mathrm{S}}$ are respectively the Poole-Frenkel lowering and Schottky coefficients. Theoretical values of these coefficients are given by $\beta_{\mathrm{PF}}=2 \beta_{\mathrm{S}}=\left(\mathrm{e}^{3} / \pi \varepsilon\right)^{1 / 2}$ and are thus $\beta_{\mathrm{PF}}=4.02 \times 10^{-5} \mathrm{eV} \mathrm{m}^{1 / 2} \mathrm{~V}^{-1 / 2}$ and $\beta_{\mathrm{S}}=2.01 \times 10^{-5} \mathrm{eV} \mathrm{m}^{1 / 2} \mathrm{~V}^{-1 / 2}$.

The values of the Schottky barrier widths and barrier heights were calculated from the slops and intercepts of Fig. 5. The values of derived parameters were found to be $w=20 \mathrm{~nm}$ and $\varphi_{\mathrm{S}}=1.17 \mathrm{eV}$ for the low voltage region, $w=320 \mathrm{~nm}$ and $\varphi_{\mathrm{S}}=1.00 \mathrm{eV}$ for the high voltage region. The values are close to those reported previously $[1,3]$. The barrier height $1.17 \mathrm{eV}$ agrees well with that determined from thermoionic emission Eqs. (2) and (3).

\section{References}

[1] A. Alımed, R.A. Collins, Phys. Status Solidi A 126, 411 (1991).

[2] A.K. Hassan, R.D. Gould, Int. J. Electron. 69, 11 (1990).

[3] T.G. Abdel-Malik, R.M. Abdel Latif, Physica B 205, 59 (1995).

[4] R.O. Loutfy, Phys. Status Solidi A 65, 659 (1981).

[5] N. Minami, K. Sasaki, K. Tsuda, J. Appl. Phys. 54, 6765 (1983).

[6] T.G. Abdel-Malik, A.A. Ahmed, A.S. Riad, Phys. Status Solidi A 121, 507 (1990). 
[7] C.R. Westgate, G. Warfield, J. Chem. Phys. 46, 94 (1967).

[8] H. Koezuka, Etoh, J. Appl. Phys. 54, 2511 (1983).

[9] B. Boudjema, G. Guiloud, M. Gamoudi, M. Maitot, J.J. Andre, M. Martin, J. Simon, J. Appl. Phys. 56, 2323 (1984).

[10] S.M. Sze, Semiconductor Devices, Physics and Technology, Wiley, New York 1985.

[11] H. Norde, J. Appl. Phys. 50, 5052 (1979).

[12] K. Sato, Y. Yasumura, J. Appl. Phys. 58, 3655 (1985).

[13] S.K. Cheung, N.W. Cheung, Appl. Phys. Lett. 49, 58 (1986).

[14] R.D. Gould, M.S. Ralıman, J. Phys. D 14, 79 (1981).

[15] R.D. Gould, Thin Solid Films 125, 63 (1985).

[16] M.A. Lampert, Rep. Prog. Phys. 27, 329 (1964).

[17] J.G. Simmons, J. Phys. D 38, 2738 (1971). 